\title{
CONGENITAL AND ACQUIRED ANOMALIES OF THE CAUDAL VERTEBRAE IN DOGS: RADIOGRAPHIC CLASSIFICATION AND PREVALENCE EVALUATION
}

\author{
Michaela PANINÁRovÁ ${ }^{1 *}$, Ladislav STEHLíK ${ }^{1}$, Pavel PROKS ${ }^{1}$ and Massimo VIGNOLI ${ }^{2}$ \\ ${ }^{1}$ Department of Diagnostic Imaging, Small Animal Clinic, Faculty of Veterinary Medicine, \\ University of Veterinary and Pharmaceutical Sciences Brno, Palackého tr. 1946/1, \\ Brno 612 42, Czech Republic; ${ }^{2}$ Faculty of Veterinary Medicine, University of Teramo, \\ Teramo, Italy
}

(Received 28 January 2016; accepted 4 May 2016)

\begin{abstract}
A prospective study to create radiographic classification of caudal vertebral anomalies in dogs was performed and the prevalence of the disorders estimated. Radiographic examination of the caudal vertebrae was performed in 595 client-owned dogs undergoing routine radiological examination of the hip joints. Anomalies of the caudal vertebrae were divided into four groups: (1) congenital anomalies with aberrant fusion of the vertebral epiphysis, like hemivertebra, block or transitional vertebra; (2) morphological anomalies like vertebra curva and dysplastic vertebral epiphysis; (3) post-traumatic changes, represented by fracture or luxation; and (4) degenerative changes, represented by spondylosis, osteophyte formation and mineralisation of intervertebral spaces. A total of 303 caudal vertebral anomalies were detected in 259 dogs ( $43.53 \%$ of all dogs examined). A single type of vertebral anomaly was present in 215 dogs and a combination of two pathologies was recorded in 44 dogs, which represents $83.01 \%$ and $16.99 \%$, respectively, of the affected dogs. Congenital anomaly was present in $11.09 \%$ of the dogs examined. Sacrocaudal transitional vertebra was the most common congenital anomaly, present in $12.74 \%$ of the affected dogs. Radiographic examination of the caudal vertebrae is recommended as an essential part of any screening process to confirm the presence of congenital anomaly as a cause of kinked tail.
\end{abstract}

Key words: Tail, caudal vertebra, hemivertebra, block vertebra, kink tail

Congenital spinal malformations are commonly identified in small animals and may lead to pain, myelopathy, radiculopathy, and gross spinal deformities. However, many spinal malformations do not produce overt neurological dysfunction, and it is a responsibility of the clinician to investigate and determine whether the malformation is an incidental finding or an underlying cause of clinical signs (Westworth and Sturges, 2010). Classifications of congenital anomalies and their prevalence in dogs, cats, rabbits and ferrets exist (Morgan,

*Corresponding author; E-mail: paninarovam@gmail.com; Phone: 0039 (333) 810-7758 
1968; Newitt et al., 2008; Gutierrez-Quintana et al., 2014; Mäkitaipale et al., 2015; Proks et al., 2015). Many anomalies, both in terms of type and number, are explained by the manner in which vertebral bodies form (Morgan, 1968). The numbers of vertebrae in a vertebral column and the number of independently developing secondary ossification centres contribute to this variability (Morgan, 1968). Caudal vertebrae constitute an integral part of the vertebral column and the presence of congenital anomalies in this part of the vertebral column is common (Schawalder et al., 2010).

The average number of caudal vertebrae in dogs is usually 20 , although this may vary from 6 to 23. The number of caudal vertebrae is subject to greater variation than that of vertebrae in any other region, but it may remain constant in a given breed. The cranial vertebrae tend to conform most typically to the morphologically representative type, whereas the caudal segments are gradually reduced to simple rods. The diaphysis of the caudal vertebra is completely ossified after birth. The epiphysis ossifies 2-8 weeks after birth. Complete closure of the caudal vertebra epiphysis takes place at 14 months of age (Evans and de Lahunta, 2013).

Congenital and acquired caudal vertebral malformations are clinically manifested as kink tail, stump deformation or a palpable bulge in the course of the tail. However, many of the malformations are clinically silent. Those malformations that are not associated with deviation of the long axis of the caudal spine may be visualised only radiographically (Schawalder et al., 2010).

The clinical relevance of the abnormalities and their impact on the health status of the patient is insignificant in most cases. Only a limited number of related clinically significant diseases appear in the literature. Severe neural tube defects have been documented in cats with absence of the caudal vertebrae. The Manx breed is among the most commonly affected, due to stumpy tail deformation or complete taillessness (Deforest and Basrur, 1979). Intervertebral disc protrusion or extrusion at the $\mathrm{Cd} 1-\mathrm{Cd} 2$ disk space has been described in four dogs (Lawson et al., 2011). Caudal intervertebral disk herniation should be considered as a differential diagnosis for dogs with caudal vertebral pain, pain on tail manipulation, pain during defecation, or abnormal tail carriage (Lawson et al., 2011).

An association between the presence of congenital pathology in the caudal vertebrae with the presence of congenital abnormality in another part of vertebral column may be suspected (Newitt et al., 2008; Schawalder et al., 2010; GutierrezQuintana et al., 2014).

'Kink tail' is an issue much discussed in the breeders' community, although the term may cover a number of morphologically unrelated vertebral anomalies (Schawalder et al., 2010). Its presence could be a reason to exclude an individual dog from breeding programmes.

A classification of caudal vertebral anomalies based on radiological appearance has been published by Schawalder et al. (2010) in German language. 
PANINÁROVÁ et al.

The authors of that paper divide the abnormalities into 11 groups based on radiological manifestation and aetiology but they do not report prevalence (Schawalder et al., 2010). To the best of the present authors' knowledge, no account of the prevalence of caudal vertebral anomalies has yet been published.

The authors of the current contribution assumed that the aetiology of caudal vertebral pathologies is similar to that of abnormalities in other parts of the vertebral column. The goal of this prospective study was to determine the prevalence and morphological features of caudal vertebral abnormalities in a large sample of dogs in the Czech Republic.

\section{Materials and methods}

The University of Veterinary and Pharmaceutical Sciences Brno gave ethical approval for the study. All of the owners of the dogs examined gave their informed consent to the participation of their animals in the study.

Animals

The study was conducted prospectively from September 2012 to May 2015. It included dogs undergoing radiological examination of the hip joints under intravenous sedation or intravenous anaesthesia for the purpose of dysplasia screening. Dogs with cropped tails and screw-tailed breeds such as the French Bulldog, English Bulldog and Pug were excluded.

\section{Method}

For intravenous sedation, medetomidine (10-20 $\mu \mathrm{g} / \mathrm{kg}$ i.v. Domitor, Pfizer) and butorphanol $(0.2 \mathrm{mg} / \mathrm{kg}$ i.v. Butomidor, Richter Pharma AG, Austria) were used. Medetomidine (10-20 $\mu \mathrm{g} / \mathrm{kg}$ i.v. Domitor, Pfizer), butorphanol $(0.2 \mathrm{mg} / \mathrm{kg}$ i.v. Butomidor, Richter Pharma AG) and propofol $(10 \mathrm{mg} / \mathrm{kg}$ i.v. Norofol, Norbrook, Norway) were employed for intravenous anaesthesia. Two orthogonal views of the entire caudal spine were obtained in all subjects, in laterolateral and ventrodorsal projection. All images were obtained using an X-ray machine (Proteus XR/a, GE Healthcare, Wisconsin, USA) with cassettes for computed radiography. Table-top technique was employed, without the use of a grid. The digital radiographs were stored in DICOM format using the Capsula XL (Fuji, Japan) computed radiography system. The resolution of the radiographs was $1760 \times$ 2140 and $1576 \times 1976$.

\section{Radiograph interpretation}

DICOM images of all the caudal vertebrae were reviewed. A single observer (MP) performed this in order to reduce inter-operator variability. Anoma- 
lies of the caudal vertebrae were divided into four groups: (1) congenital anomalies (Figs 1, 2 and 3); (2) morphological anomalies (Figs 4 and 5); (3) posttraumatic changes (Fig. 6); and (4) degenerative changes (Fig. 7). The prevalence of all groups of anomalies was determined.

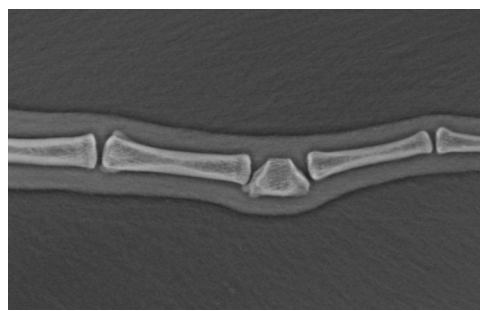

Fig. 1. Laterolateral projection of the caudal vertebrae. Hemivertebra (congenital anomalies, Group 1)

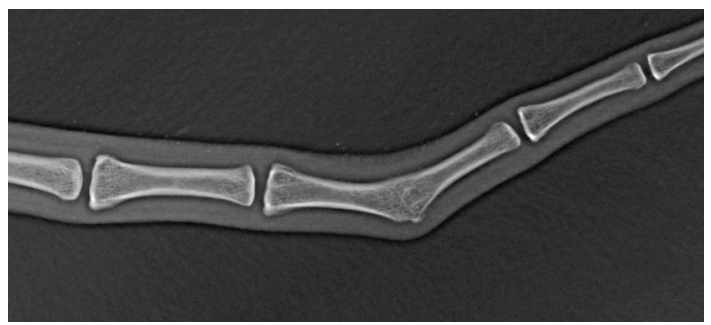

Fig. 2. Laterolateral projection of the caudal vertebrae. Block vertebra (congenital anomalies, Group 1)

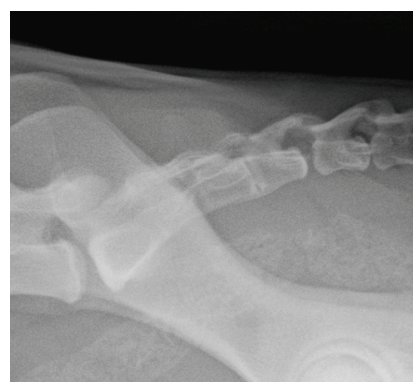

Fig. 3. Laterolateral projection of the sacrum and caudal vertebrae. Sacrocaudal transitional vertebra (congenital anomalies, Group 1). Fusion of Cd1 to the sacrum is visible

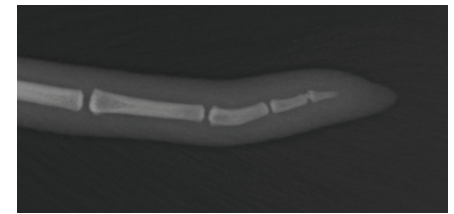

Fig. 4. Laterolateral projection of the caudal vertebrae. Curved vertebra (morphological anomalies, Group 2) 


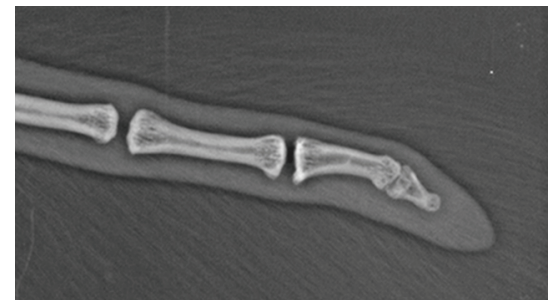

Fig. 5. Laterolateral projection of the caudal vertebrae. Epiphyseal dysplasia (morphological anomalies, Group 2)

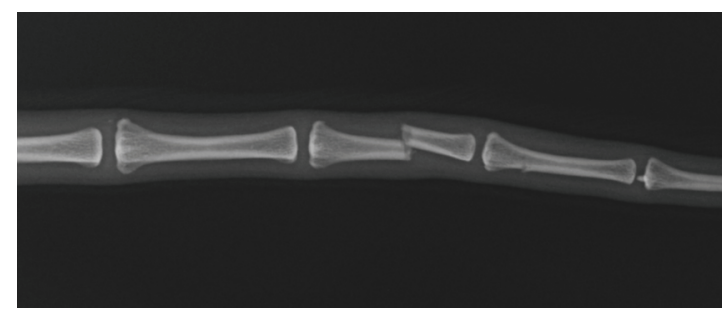

Fig. 6. Ventrodorsal projection of the caudal vertebrae. Fracture (post-traumatic changes, Group 3) in the diaphysis of the caudal vertebra

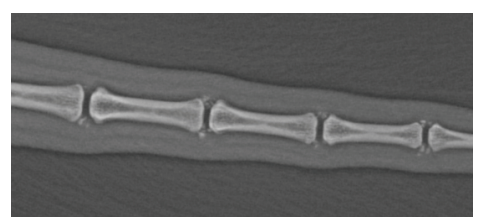

Fig. 7. Ventrodorsal projection of the caudal vertebrae. Multiple mineralisation (degenerative changes, Group 4) is visible within the intervertebral spaces

\section{Data and statistical analysis}

Chi-square analysis was used to determine differences between the sexes, with $\mathrm{P}<0.05$ considered significant. A one-sample proportion test was used for comparison of the prevalence of congenital anomalies in breeds with more than 10 dogs and total prevalence. Commercial statistical software was used to perform the analysis (Minitab 17, Minitab Inc., Coventry, UK).

\section{Results}

A total of 595 client-owned dogs presented at the Small Animal Clinic of the University of Veterinary and Pharmaceutical Sciences Brno, matched the criteria for the study. The dogs ranged in age from 4 months to 12 years (median age 2.3 years), while 303 (50.9\%) were males and 292 (49.1\%) females. No gen- 
der association emerged between the groups of vertebral anomalies $(\mathrm{P}=0.864)$. A total of 93 breeds were included. The breeds with a minimum of 10 dogs per breed were listed separately and the prevalence of congenital abnormalities was calculated separately for them. The remaining 78 breeds, with fewer than 10 dogs per breed, were classified as 'other breeds'. No statistically significant difference in the prevalence of congenital abnormalities of the caudal vertebrae was detected in any of the breeds in comparison with the prevalence in all dogs examined ( $\mathrm{P}>0.05$ for each breed, Table 1). Normal caudal vertebrae were recorded in 336 dogs $(56.47 \%)$. A total of 303 congenital and acquired anomalies were present in 259 dogs $(43.53 \%$, Table 2$)$. A single type of vertebral anomaly was revealed in $215 \operatorname{dogs}(83.01 \%)$. A combination of two types of pathology was found in 44 dogs $(16.99 \%)$. No combination of more than two types of vertebral pathology was detected. Congenital anomalies with aberrant fusion of vertebral epiphysis, represented by hemivertebra, block vertebra or transitional vertebra at the sacrocaudal junction, were present in 66 dogs $(11.09 \%$ of all examined dogs, Figs 1, 2 and 3). Morphological anomalies of the caudal vertebrae, represented by vertebra curva or dysplastic vertebral epiphysis, were present in 32 dogs (5.38\% of all examined dogs, Figs 4 and 5). Post-traumatic changes, represented by fracture, formation of callus or luxation, were detected in 45 dogs (7.56\%, Fig. 6). Degenerative changes of the caudal vertebrae and intervertebral spaces, including spondylosis, osteophyte formation and mineralisation of intervertebral spaces, were detected in 160 dogs $(26.89 \%$ of examined dogs, Fig. 7). The prevalence was calculated as the sum of single and combined pathologies. The prevalence of each type of congenital and morphological anomaly was calculated separately for each group (Table 3 ).

A high variability in morphological appearance was noticed in the last caudal vertebrae, largely in terms of shape, size, and presence of intervertebral space. The number of vertebrae with morphological variability differed between breeds of the dogs evaluated. Short and elongated vertebrae in this part of caudal spine, radiographically similar to hemivertebra (Fig. 8A) and block vertebra (Fig. $8 \mathrm{~B}$ ), were recorded, but such vertebrae were not classified as congenitally abnormal.

\section{Discussion}

The clinical manifestation of kink tail is one of the reasons for excluding a dog from breeding. In the Czech Republic, a number of breeds, including Rhodesian Ridgeback, Hovawart, Bernese Mountain Dog, White Swiss Shepherd Dog, and Dachshund, are excluded from breeding if kink tail is detected on palpation. However, it is not possible to distinguish between the various types of pathology of the caudal vertebrae by palpation alone. Identification of the pathology that has led to bending of the tail is the first step in evaluating clinical significance 
and in establishing a reasonable and evidence-based breeding regulation. Responsibility for which abnormalities should be excluded remains with the breeding clubs, but veterinarians are frequently involved in the decision-making process centring upon the aetiology of kink tail. Radiographic examination of the entire caudal spine in two orthogonal projections is essential to determine the type of abnormality.

\section{Table 1}

Prevalence of congenital anomalies in various breeds of $\operatorname{dog}(n=595)$

\begin{tabular}{lccr}
\hline \multicolumn{1}{c}{ Breed } & $\mathrm{n}^{*}$ & Cong $^{\dagger}$ & Cong $^{\ddagger}{ }^{\ddagger}$ \\
\hline German Shepherd & 77 & 10 & 12.99 \\
Rhodesian Ridgeback & 51 & 6 & 11.76 \\
White Swiss Shepherd Dog & 41 & 3 & 7.32 \\
Labrador Retriever & 31 & 1 & 3.23 \\
Golden Retriever & 19 & 0 & 0.00 \\
Belgian Shepherd Dog & 17 & 3 & 17.65 \\
Rottweiler & 16 & 0 & 0.00 \\
Leonberger & 15 & 0 & 0.00 \\
Samoyed & 15 & 0 & 0.00 \\
Border Collie & 14 & 0 & 0.00 \\
Bernese Mountain Dog & 14 & 2 & 14.29 \\
Hovawart & 14 & 2 & 14.29 \\
Czechoslovakian Wolf-dog & 12 & 0 & 0.00 \\
Alaskan Malamute & 12 & 1 & 8.33 \\
Other breeds & 247 & 38 & 15.38 \\
\hline
\end{tabular}

${ }^{*}$ n: number; ${ }^{\dagger}$ Cong: number of dogs with congenital anomalies; ${ }^{\ddagger}$ Cong\%: percentage of dogs with congenital anomalies

Table 2

Prevalence of caudal vertebral anomalies

\begin{tabular}{|c|c|c|c|}
\hline Anomalies & $\mathrm{n}^{*}$ & Pop $\%^{\dagger}$ & Anm $\%{ }^{\ddagger}$ \\
\hline \multicolumn{4}{|c|}{ Single anomalies } \\
\hline Congenital (Group 1) & 45 & 7.56 & 17.38 \\
\hline Morphological (Group 2) & 19 & 3.19 & 7.34 \\
\hline Post-traumatic (Group 3) & 29 & 4.87 & 11.20 \\
\hline Degenerative (Group 4) & 122 & 20.50 & 47.10 \\
\hline \multicolumn{4}{|c|}{ Combined anomalies } \\
\hline Congenital (Group 1) + morphological (Group 2) & 2 & 0.34 & 0.77 \\
\hline Congenital (Group 1) + post-traumatic (Group 3) & 4 & 0.67 & 1.54 \\
\hline Congenital (Group 1) + degenerative (Group 4) & 15 & 2.52 & 5.79 \\
\hline Morphological (Group 2) + degenerative (Group 4) & 11 & 1.85 & 4.25 \\
\hline Post-traumatic (Group 3) + degenerative (Group 4) & 12 & 2.02 & 4.63 \\
\hline Total & 259 & 43.53 & 100.00 \\
\hline
\end{tabular}

${ }^{*} \mathrm{n}$ : number; ${ }^{\dagger}$ Pop\%: percentage of population $(\mathrm{n}=595) ;{ }^{\ddagger} \mathrm{Anm} \%$ : percentage of dogs affected $(\mathrm{n}=259)$ 
Table 3

Prevalence of specific types of anomalies from Group 1 and Group 2

\begin{tabular}{lccc}
\hline Congenital (Group 1) & $\mathrm{n}^{*}$ & Pop\% & Anm\% ${ }^{\ddagger}$ \\
\hline Hemivertebra & 18 & 3.03 & 6.95 \\
Block vertebra & 15 & 2.52 & 5.79 \\
Transitional vertebra & 33 & 5.55 & 12.74 \\
Total & 66 & 11.09 & 25.48 \\
\hline Morphological (Group 2) & $\mathrm{n}^{*}$ & Pop\% $\%^{\dagger}$ & $\mathrm{Anm} \%^{\ddagger}$ \\
\hline Curved vertebra & 18 & 3.03 & 6.95 \\
Epiphyseal dysplasia & 14 & 2.35 & 5.41 \\
Total & 32 & 5.38 & 12.36 \\
\hline
\end{tabular}

${ }^{*} \mathrm{n}$ : number; ${ }^{\dagger} \mathrm{Pop} \%$ : percentage of population $(\mathrm{n}=595) ;{ }^{\star} \mathrm{Anm} \%$ : percentage of dogs affected $(\mathrm{n}=259)$
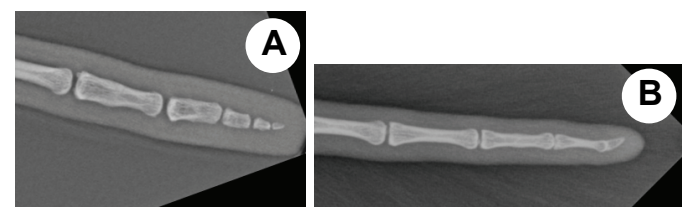

Fig. 8. Laterolateral projection of the last caudal vertebrae. High variability of morphological appearance can be observed. Short (A) and elongated vertebrae (B) in this part of the caudal spine, radiographically similar to hemivertebra and block vertebra, respectively, are presented

In the present study, abnormalities of the caudal vertebrae were divided into four groups on the basis of radiological presentation. An attempt was made to simplify and reduce the large number of groups in a previously published classification that divided pathologies into eleven categories. In that study, not all the groups of abnormalities were based on solely radiological manifestation, but also on hypotheses of probable aetiology (Schawalder et al., 2010). The main focus of the current study was upon the possibility of distinguishing congenital abnormalities (hemivertebra, block vertebra, transitional vertebra) from morphological, post-traumatic and degenerative changes. The capacity to distinguish between congenital and other causes of kink tail is essential in the selection of dogs to be excluded from breeding. It proved possible to determine each type of pathology on the basis of its radiological manifestation in the greater part of the caudal vertebrae, while it was not possible to distinguish congenital anomalies and variability from normal anatomy in only the caudal part of the tail. The reduction of the vertebral anatomy to rudimentary bone rods is the main source of this difference. Further investigation of the apical part of the tail is required to demonstrate the presence of congenital abnormalities. Radiographic examination of the caudal 
vertebrae in juvenile animals, when the physis is open, is recommended to determine the presence of hemivertebra or block vertebra.

In the present study, transitional vertebra at the sacrocaudal junction was the most common congenital anomaly, presenting in $12.74 \%$ of affected dogs. This is in agreement with the existing literature, in which the sacrocaudal junction emerged as the region with the greatest prevalence of vertebral anomaly, present in $27.10 \%$ of affected dogs (Morgan, 1968). The sacrocaudal junction has been reported as the most commonly affected part of the vertebral column in cats as well. According to Newitt et al. (2008) it was present in $12 \%$ of the affected cats.

It is suspected that hemivertebra, block vertebra and transitional vertebra in the caudal vertebral column are heritable (Schawalder et al., 2010). No information about family relations was available for the current study. Moreover, there were no radiographs of the whole vertebral column in the animals with congenital vertebral anomalies. Thus, no conclusion can be reached here on heritability or association between vertebral anomalies in the caudal vertebrae and the presence of anomalies in other parts of vertebral column. The low numbers of dogs representing each breed (maximum 77) and the wide variability of breeds (93 of them) presented in the study meant that the results of the statistical test disclosed no significant difference in prevalence among breeds. However, the Belgian Shepherd Dog, Bernese Mountain Dog and Hovawart had the highest prevalence of congenital anomalies in the caudal vertebrae, and it may be speculated that heritability, or at least familiar predisposition, exists in these breeds.

The brachycephalic breeds were excluded from this study, since the presence of screw tail is characteristic of them. A high number of congenital pathologies, in the form of multiple hemivertebra and block vertebra, have been documented (Done et al., 1975; Gutierrez-Quintana et al., 2014). The aim of the present study was to determine the prevalence of anomalies in breeds for which the presence of congenital anomalies in the caudal vertebrae is not typical. Dogs with crop tail were excluded because some of the caudal vertebrae are missing in them and this could lead to false negative results.

In conclusion, the classification presented in the study, based on radiological manifestation of abnormalities, should provide veterinarians a tool to help distinguish between congenital and acquired malformations of the caudal vertebrae. Palpation of the tail as a method of determining the presence of congenital abnormality in kink tail is inadequate. This technique is questionable if breeding clubs wish to exclude dogs with clinical manifestation of kink tail, or dogs with congenital abnormality, from breeding. If congenital anomalies are to be excluded, then radiographic examination of the caudal vertebrae is an essential part of screening process. 


\section{References}

Deforest, M. E. and Basrur, P. K. (1979): Malformations and the manx syndrome in cats. Can. Vet. J. 20, 304-314.

Done, S., Drew, R., Robins, G. and Lane, J. (1975): Hemivertebra in the dog: Clinical and pathological observations. Vet. Rec. 96, 313-317.

Evans, H. E. and de Lahunta, A. (2013): The skeleton. In: Evans, H. E. and de Lahunta, A. (eds) Miller's Anatomy of the Dog. Elsevier, St. Louis. pp. 80-157.

Gutierrez-Quintana, R., Guevar, J., Stalin, C., Faller, K., Yeamans, C. and Penderis, J. (2014): A proposed radiographic classification scheme for congenital thoracic vertabral malformations in brachycephalic 'screw-tailed' dog breeds. Vet. Radiol. Ultrasound 55, 585-591.

Lawson, C. M., Reichle, J. K., McKlveen, T. and Smith, M. O. (2011): Imaging findings in dogs with caudal intervertebral disc herniation. Vet. Radiol. Ultrasound 52, 487-491.

Mäkitaipale, J., Harcourt-Brown, F. M. and Laitinen-Vapaavuori, O. (2015): Health survey of 167 pet rabbits (Oryctolagus cuniculus) in Finland. Vet. Rec. 177, 418.

Morgan, J. P. (1968): Congenital anomalies of the vertebral column of the dog: A study of the incidence and significance based on a radiographic and morphologic study. Vet. Radiol. 9, 21-29.

Newitt, A., German, A. J. and Barr, F. J. (2008): Congenital abnormalities of the feline vertebral column. Vet. Radiol. Ultrasound 49, 35-41.

Proks, P., Stehlik, L., Paninarova, M., Irova, K., Hauptman, K. and Jekl, V. (2015): Congenital abnormalities of the vertebral column in ferrets. Vet. Radiol. Ultrasound 56, 117-123.

Schawalder, P., Dietschi, E. and Stich, H. (2010): Congenital and acquired malformations of coccygeal vertebrae in dogs [in German]. Wien. Tierärztl. Monatsschr. 97, 185-202.

Westworth, D. R. and Sturges, B. K. (2010): Congenital spinal malformations in small animals. Vet. Clin. North Am. Small Anim. Pract. 40, 951-981. 